Conclusions The results suggest that in obese children undergoing nutritional interventions longitudinal changes in liver fat content may be associated with change in serum transaminases suggesting novelty in monitoring NAFLD in childhood obesity.

\section{COMPARISON BETWEEN OVERWEIGHT/OBESE (OW/OB) PATIENTS: COELIAC VS NON COELIAC ONES}

doi:10.1136/archdischild-2012-302724.0711

R Mercurio, D Guttadoro, M Podagrosi, A Grimaldi, M Barbato, AM Caiazzo, L Petrarca, A Vania. Dept of Paediatrics and Paediatric Neuro-Psychiatry, 'Sapienza' University of Rome, Rome, Italy

Background and Aims In literature there are case reports of coeliac disease (CD) diagnosed in $O W / O B$ children and studies assessing the body weight excess (EBW) at CD diagnosis. Besides, an incorrect gluten-free-diet (GFD) often leads to EBW. Aim of this study is to assess the effect of a balanced diet in coeliac patients (CP) with EBW compared to matched non-coeliac patients (NCP) with EBW.

Methods and results: In 2006-2010, we selected 8 (4M, 4F, age $=11.35 \pm 3.79$ ) out of $29 \mathrm{CP}$ we follow; they all had EBW $(z-B M I=1.93 \pm 0.69)$ and were compliant to the GFD. Follow-up lasted $11.62 \pm 1.68$ months, with a $z$-BMI reduction in all cases (z-BMI=1.45 \pm 1.01$)$. Among the matched 8 NCP (4M, 4F, age $=10.52 \pm 3.60$; start-up's $z-B M I=2.07 \pm 0.59$;), followed-up for the same period of time, 7 reduced their $z-B M I(1.57 \pm 0.67)$, while in 1 it worsened.

Conclusions Despite the limited number of patients, our study underlines the importance of a strict supervision in CP with EBW to improve their nutritional status. We also noticed that the coherent adhesion to a balanced GFD and the consequent effect on z-BMI are better than those among NCP, probably because in CP the nutritional prescriptions are perceived as indispensable for the therapy of CD.

\section{Bibliografia:}

Mariani P et al. J Pediatr Gastroenterol Nutr 1998; 27:519-523;

Polito C et al. Nutr Res 1992; 12:353-358;

Rea F et al. J Pediatr Gastroenterol Nutr 1996; 23:408-412;

Dickey W, Kearney N. Am J Gastroenterol 2006; 101:2356-2359;

Aydogdu S et al. Dig Dis Sci 2009; 54:2183-2187;

Patro B, Szajewska H. Curr Opin Clin Nutr Metab Care. 2010; 13:300-4

\section{SAFETY AND EFFICACY OF OMEGAVEN IN PRETERM NEONATES WITH PARENTERAL NUTRITION ASSOCIATED LIVER DISEASE}

doi:10.1136/archdischild-2012-302724.0712

${ }^{1} \mathrm{G}$ Booth, 'T Havranek, 'E Armbrecht, ${ }^{2} \mathrm{M}$ Revenis, ${ }^{2} \mathrm{C}$ Klein, ${ }^{2} \mathrm{~L}$ Scavo. ${ }^{1}$ Saint Louis University, St. Louis, MO; ${ }^{2}$ George Washington Univ., Children's National Med Center, Washington, DC, USA

Background Omegaven, a fish oil derived parenteral lipid emulsion rich in omega-3 fatty acids, has been used in the United States under Investigational New Drug applications to treat parenteral nutrition associated liver disease (PNALD) in neonates.

Aims To evaluate the safety profile and changes in serum direct bilirubin levels in preterm neonates with moderate to severe PNALD treated with Omegaven

Methods A prospective observational study of parenteral nutrition (PN) dependant neonates with consecutive serum direct bilirubin levels above $3 \mathrm{mg} / \mathrm{dL}$, separated by a minimum of 7 days, who were expected to require PN for at least 3 more weeks were considered for Omegaven therapy. Eligible neonates were treated with Omegaven, as the only lipid infusion, at a maximum rate of $1 \mathrm{~g} / \mathrm{kg} /$ day. Omegaven therapy was stopped once the serum direct bilirubin level was less than $1 \mathrm{mg} / \mathrm{dL}$ on consecutive weeks, or the patient tolerated adequate enteral nutrition to stop PN. The change in serum direct bilirubin was compared using the Wilcoxon signedrank test.

Results 17 neonates were eligible for the analysis with a mean gestational age at birth of 26.6 weeks and mean birth weight of $911 \mathrm{~g}$. Omegaven was administered for a median of 57 days. The neonates tolerated Omegaven well, without significant changes noted in the safety indicators (coagulation profile, platelet count, serum triglyceride, glucose, albumine).

Serum direct bilirubin levels decreased significantly during the treatment course $(p=0.035)$.

Conclusions Significant decrease in serum direct bilirubin levels was observed in neonates with PNALD; omegaven was well tolerated.

\section{COMPARISON BETWEEN PROPOFOL VERSUS PROPOFOL- KETAMINE COMBINATION IN PAEDIATRIC UPPER GASTRO- INTESTINAL ENDOSCOPY}

doi:10.1136/archdischild-2012-302724.0713

${ }^{1} 0$ Genovese, ${ }^{2} \mathrm{~A}$ Chiaretti, ${ }^{1} \mathrm{~A}$ Mancino, ${ }^{2} \mathrm{~A}$ Gatto. ${ }^{1}$ Paediatric Intensive Care Unit; 2Pediatrics, Policlinico Gemelli University Hospital, Roma, Italy

Objective To assess the safety and effectiveness of the ketamine plus propofol combination versus propofol alone for procedural sedation in pediatric patients undergoing upper GI endoscopy.

Patients and design: A retrospective case series of patients undergoing procedural sedation for upper GI endoscopy was studied. Median age was $6.3(0.4-13.1)$ years. 29 patients received propofol alone while 37 patients received propofol plus ketamine; the child's degree of sedation was scored using the modified Ramsay sedation scale. Procedural success (length of the procedure), adverse effects, recovery time, and vital signs were also measured.

Results No patient in either group experienced major adverse events or required tracheal intubation. In group receiving ketamine and propofol, the total dose of propofol required was significantly lower than in Propofol alone (3.4. $\pm 3.2 \mathrm{mg} / \mathrm{kg}$ vs. $5.2 \pm 3.4 \mathrm{mg} / \mathrm{kg}$; $\mathrm{P}<0.001)$. The incidence of hypotension was also significantly lower (10\% vs. $37 \%$; $\mathrm{P}<0.001)$. Both best analgesia and shorter recovery time were obtained with the propofol-ketamine association. No differences were observed in the degree of sedation and in the awakening quality score between the two groups.

Conclusions The combination of propofol and ketamine provides a good solution for procedural sedation in during GI endoscopic procedures. Compared to propofol alone, ketamine and propofol results in less hypotension and reduces the incidence of respiratory depression.

\section{RELATIONSHIP BETWEEN ESOPHAGEAL SWALLOW PATTERNS AND REFLUX CHARACTERISTICS IN NEWBORNS WITH SYMPTOMS OF GASTROESOPHAGEAL REFLUX DISEASE}

doi:10.1136/archdischild-2012-302724.0714

${ }^{1} \mathrm{~F}$ Cresi, ' $\mathrm{SA}$ Liguori, ${ }^{\mathrm{E} E}$ Locatelli, 'E Maggiora, ${ }^{2} \mathrm{~F}$ Savino, 'E Bertino. ${ }^{1}$ Neonatal Intensive Care Unit, Department of Paediatrics; ${ }^{2}$ Dep. of Pediatrics, University of Torino, Torino, Italy

Background and Aims Transient lower esophageal sphincter relaxations are primarily responsible for GER in healthy infants and plays an important role in preterm and term infants with GER disease (GERD). Gastroesophageal dismotility due to immaturity could also promote impaired swallowing patterns influencing the esophageal clearance. The aim of this study was to investigate the relationship between esophageal swallow patterns and reflux characteristics in newborns with GERD symptoms. 
Methods Esophageal swallow patterns and refluxes were visually evaluated in preterm and at term bottle fed newborns who underwent Multichannel Intraluminal Impedance (MII) analysis for clinical suspect of GERD.

The swallow frequency, the mean Bolus Head Advance Time (BHAT), the mean Total Bolus Transit Time (TBTT) and reflux characteristics (frequency, duration and impedance bolus exposure index) were assessed for each patient.

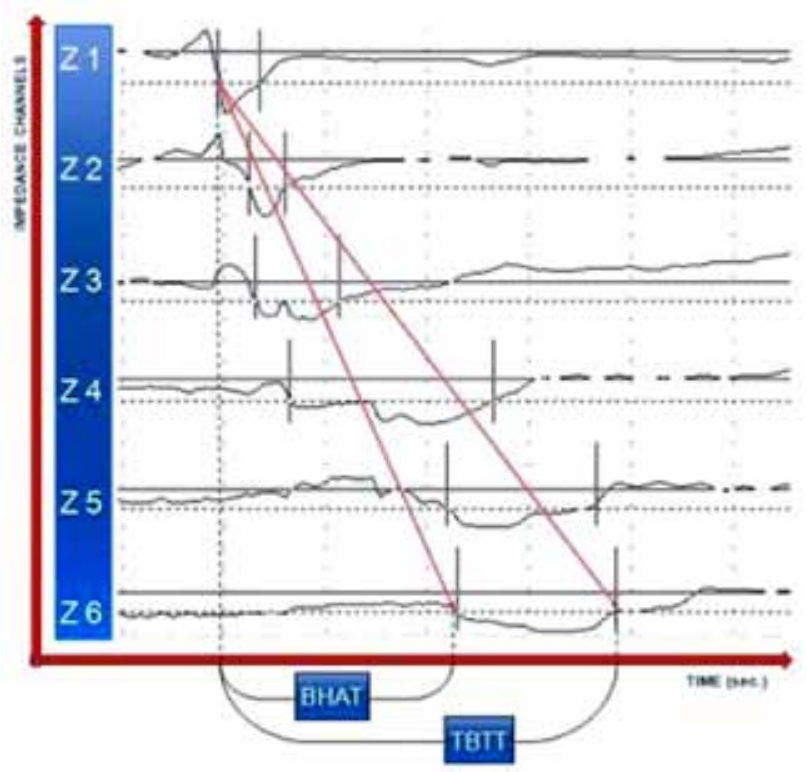

Abstract 714 Figure 1

Pearson correlation was used to evaluate the relationship between swallow and reflux variables; $p$ was set at 0.05 .

Results Fifty-four newborns (10 preterm) were enrolled in the study. They had a median(IOR) age of 22(22.5) days, a Gestational age of 37(6) weeks and a postconceptional age of 41.8(4.9) weeks. A significant positive correlation was found between TBTT and IBEI $\%(r=0.494 ; p<0.001)$ and between TBTT and reflux duration $(\mathrm{r}=0.321 ; \mathrm{p}=0.018)$.

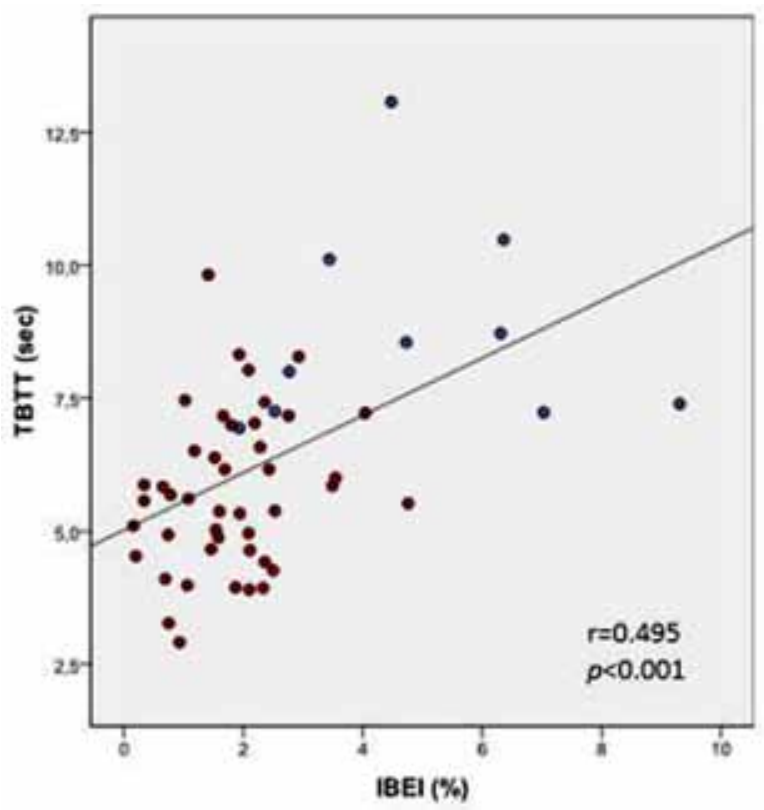

Abstract 714 Figure 2
Conclusions MII is a valid technique to evaluate esophageal bolus transit and refluxes. Our data support that esophageal motor dysfunction due to immaturity affect esophageal swallowing patterns and esophageal clearance time in GERD newborns.

\section{DENTAL EROSION AND GASTROESOPHAGEAL REFLUX DISEASE (GERD) IN CHILDREN}

doi:10.1136/archdischild-2012-302724.0715

${ }^{1} \mathrm{~F}$ Javadzadeh, ${ }^{2} \mathrm{M}$ Rafeey. 'Dentistry, Tabriz University of Medical Sciences, Aras

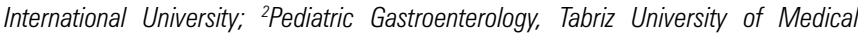
Sciences, Liver \& Gastrointestinal Research Center, Tabriz, Iran

Background Dental erosion is a complication of gastroesophageal reflux Disease (GERD) in adults; in children, it is not clear if GER has a role in dental pathologic conditions. Dietary intake, oral hygiene, high bacterial capacity, and decreased salivary flow might contribute individually to GERD development or dental erosion, but their potential involvement in dental erosion from GER is not agreed. We investigated the prevalence of dental erosion among children with and without GER symptoms, and whether salivary flow rate to location-specific dental erosion

Methods We performed a cross-sectional study of 40 children (ages,3-6 y) with symptoms of GERD and 30 asymptomatic children (controls); all completed a questionnaire on dietary exposure. Teeth were examined for erosion into dentin, erosion locations, and affected surfaces. All subjects responded a detailed frequency questionnaire related to acidic drinks, foods, and sugar consumption and participated in a clinical dental examination. The caries experience of the children was recorded according to World Health Organization criteria, and erosion was scored according to the Eccles and Jenkins grading scale.

Results This Survey showed that the prevalence of erosion on palatal surfaces of the primary teeth was $42 \%$ in 3-6-year-olds with GERD. This finding to be significantly higher than for healthy subjects $(\mathrm{P}<0.05)$. The salivary flow rate, and frequency of acidic drinks, foods, and sugar consumption were found to be similar in both groups.

Conclusion This current investigation has showed that GERD children were at an increased threat of developing erosion and caries compared with healthy subjects.

\section{DOES BLOODY ASPIRATE REFLECT THE STATE OF UPPER GASTROINTESTINAL MUCOSA IN A CRITICALLY ILL NEWBORN?}

doi:10.1136/archdischild-2012-302724.0716

R Tomerak, AA El Badawy, AE Eskander, AH Mahmoud. Cairo University, Cairo, Egypt

Background and study aims Critically ill newborns have many risk factors to develop stress related mucosal lesions (SRML). We used upper endoscopy to evaluate the presence of SRML in these neonates, to know the specificity and sensitivity of the bloody gastric aspirate to detect SRML and to identify the risk factors associated with the presence of SRML and bloody gastric aspirate.

Patients and methods This is a cross-sectional study done on 100 critically ill newborn after becoming clinically stable. SRML were diagnosed if there is hyperemia, erosions or ulcers in the esophagus, stomach, and/or the duodenum. The association between the various clinico-laboratory findings and the presence of SRML and bloody gastric aspirate were studied.

Results SRML were found in $77 \%$ of neonates in the NICU though frank bloody aspirate was detected in only $22 \%$ of neonates. The presence of bloody aspirate showed low sensitivity (24.68\%) for the presence of SRML and high specificity (86.96\%). The presence of bloody gastric aspirate showed a double fold risk for the presence SRML $(\mathrm{OR}=2.184, \mathrm{CI}=0.584-8.171)$. Factors associated with SRML 